\title{
Importance of aerosol composition and mixing state for cloud droplet ac- tivation over the Arctic pack ice in summer
}

\author{
C. Leck and E. Svensson
}

Correspondence to: C. Leck (lina@misu.su.se) 


\section{Supplementary material}

2 We developed a new implementation (written in Matlab) of the adiabatic parcel model

3 described by Pruppacher and Klett (1997). It consists of essentially the same equations as the

4 model presented in Leaitch et al. (1986), with the difference that we use the full implicit

5 dynamic diffusional growth equation for particles in each size bin:

6

$7 \quad r \frac{d r}{d t}=\frac{D^{\prime} M_{w} e_{s a t, w}}{\rho_{s} R T}\left(S-\frac{1}{1+\delta} \exp \left[\frac{L_{e} M_{w}}{R T}\left(\frac{\delta}{1+\delta}\right)+\frac{2 \mathrm{M}_{w} \sigma_{s / a}}{R T(1+\delta) \rho_{w} r_{i}}-\frac{\nu \Phi_{s} \varepsilon_{m} M_{w} \rho_{N} r_{N}^{3}}{M_{s} ?_{w}\left(r^{3}-r_{N}^{3}\right)}\right]\right)$

8 where:

$9 \quad \mathrm{r} \quad$ - radius of particle

$10 \mathrm{t} \quad-$ time

$11 \quad \mathrm{M}_{\mathrm{w}}-$ molecular weight of water

$12 \mathrm{e}_{\mathrm{sat}, \mathrm{w}}$ - saturation pressure of water vapor above a water surface

$13 \rho_{s}-$ density of the droplet solution

$14 \mathrm{R}$ - universal gas constant

$15 \mathrm{~T}$ - temperature of the gas surroundings

16 S - supersaturation

$17 \mathrm{~L}_{\mathrm{e}} \quad$ - latent heat of evaporation of water

$18 \sigma_{\mathrm{s} / \mathrm{a}}-$ surface tension at solution/air interface

$19 \rho_{\mathrm{w}}-$ density of pure water

$20 v-$ ion number of the salt

$21 \Phi_{\mathrm{s}}-$ osmotic coefficient of droplet solution

$22 \varepsilon_{\mathrm{m}}-$ mass fraction of soluble material

$23 \rho_{\mathrm{N}}-$ density of dry nucleus

$24 \mathrm{M}_{\mathrm{s}}-$ molecular mass of nucleus material 
$1 \quad \mathrm{r}_{\mathrm{N}, \mathrm{I}} \quad$ - radius of dry nucleus

2

$3 \delta=T_{d} / T-1$, where $T_{D}$ is the droplet temperature, gives a measure of the heating or

4 cooling caused by latent heat released when the droplet grows or shrinks. It is given by:

5

$6 \quad \delta=\frac{L_{e} \rho_{s}}{T K^{\prime}} r \frac{d r}{d t}$

7

8 The D' and K' are modified diffusion and thermal conduction coefficients:

$9 \quad D^{\prime}=\frac{D}{\left[\frac{r}{r+\lambda}+\frac{D}{r \alpha_{c}} \sqrt{\frac{2 \pi M_{w}}{R T}}\right.}$

10

11

$$
K^{\prime}=\frac{k}{\left[\frac{r}{r+\lambda}+\frac{k}{r \alpha_{t} \rho c_{p a}} \sqrt{\frac{2 \pi M_{A}}{R T}}\right.}
$$

12

13 where:

14 D - pressure and temperature dependent diffusivity of water vapor in air

$15 \lambda-$ constant $\left(1.2 * 10^{-7} \mathrm{~m}\right)$

$16 \alpha_{\mathrm{c}}-$ condensation accommodation coefficient

$17 \mathrm{k} \quad-$ heat conductivity

$18 \alpha_{\mathrm{t}}-$ condensation accommodation coefficient

$19 \quad \mathrm{M}_{\mathrm{A}}-$ mean molecular mass of air 
1 We simplify the Raoult term in the particle growth equation by identifying it as the water

2 activity.

3

$$
\hat{M} \Phi=-\ln \left(a_{w}\right)
$$

4

$$
\frac{\nu \Phi_{s} \varepsilon_{m} M_{w} \rho_{N} r_{N}^{3}}{M_{s} \rho_{w}\left(r^{3}-r_{N}^{3}\right)}=v M
$$

5 Recent research has shown that both of the accommodation coefficients are likely to have

6 values close to unity for pure water surfaces (e.g. Winkler et al., 2004, Winkler et. al.,

7 2006, Morita et al., 2004).

8 Due to the dependence of $\delta$ on $\mathrm{dr} / \mathrm{dt}$, the system of ordinary differential equations is implicit, 9 and hence we need to provide both starting values of $T, p, S, w$ and $r_{i}$ and their time 10 derivatives. There are a few numerical caveats in the model, such as particle sizes shrinking 11 below the dry size or even becoming negative. Unphysical developments like these are 12 avoided by artificially setting the particle size derivatives positive when the droplet size 13 approaches the size of the dry particle.

14 In contrast to the model of Leaitch et al. (1986) we make no assumptions of log-normal 15 aerosol distributions. Instead, we directly as input use the measured size distributions by the TDMPS. The lower cut-off for the chemical information is $22 \mathrm{~nm}$ in diameter, which is well below the size required to act as $\mathrm{CCN}$ in the conditions of his study. Chemical mass concentration data from LPI impactor samples are interpolated on to the TDMPS size bins and thereafter converted to number concentration assuming spherical particles.

20

$$
r_{i} \frac{d r_{i}}{d t}=\frac{D^{\prime} M_{w} e_{s a t, w}}{\rho_{s} R T}\left(S-\frac{1}{1+\delta} \exp \left[\frac{L_{e} M_{w}}{R T}\left(\frac{\delta}{1+\delta}\right)+\frac{2 \mathrm{M}_{w} \sigma_{s / a}}{R T(1+\delta) \rho_{w} r_{i}}-\frac{\nu \Phi_{s} \varepsilon_{m} M_{w} \rho_{N} r_{N, i}^{3}}{M_{s} \rho_{w}\left(r_{i}^{3}-r_{N, j}^{3}\right)}\right]\right)
$$


1 For an isolated air parcel ascending adiabatically with the vertical speed $\mathrm{V}$, the

2 temperature $\mathrm{T}$, pressure $\mathrm{p}$, supersaturation $\mathrm{S}$ and liquid water content are described by the 3 equations:

4

5

6

$7-\frac{d T}{d t}=\frac{g V}{c_{p a}}+\frac{L_{e}}{c_{p a}} \frac{d w}{d t}$

8

$9 \quad \frac{d p}{d t}=-\frac{g p V}{R_{a} T}$

10

$11 \frac{d S}{d t}=\frac{p}{\varepsilon e_{s a t, w}}-(1+S)\left\lfloor\frac{? L_{e}}{R_{a} T^{2}} \frac{d T}{d t}+\frac{g V}{R_{a} T}\right\rfloor$

12

$13 \quad \frac{d w}{d t}=\sum_{i} 4 \pi n_{i} r_{i}^{2} \frac{d r_{i}}{d t}$

14

15 In this study, however, we use $\mathrm{V}=0$ and artificially change $\mathrm{S}$ in the way the $\mathrm{CCN}$ counter 16 does.

17 